\title{
Sweet Taste Perception is Associated with Body Mass Index at the Phenotypic and Genotypic Level
}

\author{
Liang-Dar Hwang, ${ }^{1,2, *}$ Gabriel Cuellar-Partida, ${ }^{1,2, *}$ Jue-Sheng Ong, ${ }^{1,2, *}$ Paul A. S. Breslin, ${ }^{3,4}$ \\ Danielle R. Reed, ${ }^{3}$ Stuart MacGregor, ${ }^{1}$ Puya Gharahkhani, ${ }^{1}$ Nicholas G. Martin, ${ }^{1}$ and Miguel E. Rentería ${ }^{1}$ \\ ${ }^{1}$ OIMR Berghofer Medical Research Institute, Brisbane, Queensland, Australia \\ ${ }^{2}$ School of Medicine, University of Queensland, Brisbane, Queensland, Australia \\ ${ }^{3}$ Monell Chemical Senses Center, Philadelphia, Pennsylvania, USA \\ ${ }^{4}$ Department of Nutritional Sciences, School of Environmental and Biological Sciences, Rutgers University, New \\ Brunswick, New Jersey, USA
}

\begin{abstract}
Investigations on the relationship between sweet taste perception and body mass index (BMI) have been inconclusive. Here, we report a longitudinal analysis using a genetically informative sample of 1,576 adolescent Australian twins to explore the relationship between BMI and sweet taste. First, we estimated the phenotypic correlations between perception scores for four different sweet compounds (glucose, fructose, neohesperidine dihydrochalcone (NHDC), and aspartame) and BMI. Then, we computed the association between adolescent taste perception and BMI in early adulthood (reported 9 years later). Finally, we used twin modeling and polygenic risk prediction analysis to investigate the genetic overlap between BMI and sweet taste perception. Our findings revealed that BMI in early adulthood was significantly associated with each of the sweet perception scores, with the strongest correlation observed in aspartame with $r=0.09$ $(p=.007)$. However, only limited evidence of association was observed between sweet taste perception and $\mathrm{BMI}$ that was measured at the same time (in adolescence), with the strongest evidence of association observed for glucose with a correlation coefficient of $r=0.06(p=.029)$ and for aspartame with $r=0.06$ ( $p$ $=.035)$. We found a significant $(p<.05)$ genetic correlation between glucose and NHDC perception and BMI. Our analyses suggest that sweet taste perception in adolescence can be a potential indicator of BMI in early adulthood. This association is further supported by evidence of genetic overlap between the traits, suggesting that some BMI genes may be acting through biological pathways of taste perception.
\end{abstract}

Keywords: taste perception, BMI, sweetness, longitudinal analysis, genetic risk, twin study

A high body mass index (BMI) is associated with increased risk for metabolic, cardiovascular, and respiratory diseases, and several types of cancer, such as breast and colorectal cancers (Solomon \& Manson, 1997). Obesity can arise as a result of genetic predisposition (Speliotes et al., 2010), metabolic problems (Wisse, 2004), hormonal changes (Björntorp, 1995), physical activity (Fox \& Hillsdon, 2007), and/or eating behavior (Wardle, 2007). The latter is heavily influenced by cognitive factors that allow individuals to assign a subjective reward value to specific kinds of foods (Breslin \& Spector, 2008). One of these (cognitive) factors is taste perception, which is the sensory impression of food on the tongue.

Among the five basic human taste qualities (i.e., sweet, bitter, sour, salty, and umami), sweetness is generally considered pleasant at moderate intensities and favored by most individuals (Moskowitz et al., 1974). Sweetness is detected by sweet taste receptors in the oral cavity, which send a signal to the brain where taste sensation is elicited (Rolls, 2015). Sweet taste receptors are also expressed along the digestive system, including the pancreas, bladder, gastrointestinal, and adipose tissues (Laffitte et al., 2014), where they do not evoke sweet sensation but are involved in many physiological functions, including glucose homeostasis (Laffitte et al., 2014), insulin secretion (Kyriazis et al., 2014), and adipogenesis (Masubuchi et al., 2013).

Earlier studies investigating the association between BMI and sweet taste perception have presented inconsistent

RECEIVED 4 February 2016; ACCEPTEd 7 June 2016. First published online 5 August 2016.

ADDRESS FOR CORRESPONDENCE: Liang-Dar Hwang, Genetic Epidemiology Group, QIMR Berghofer Medical Research Institute, Herston QLD 4006, Australia. E-mail: Daniel.Hwang@qimrberghofer.edu.au

${ }^{*}$ These authors contributed equally to this work. 
findings. Some studies reported that obese individuals perceive the same candy or sucrose solution as less sweet than their non-obese counterparts (Bartoshuk et al., 2006; Overberg et al., 2012). However, a handful of other studies did not find direct association between BMI and sweet taste perception or sensitivity (Anderson, 1995; Grinker, 1978; Joseph et al., 2016).

Twin studies have reported that individual differences in both sweet taste perception and BMI are partially attributable to genetic variation $\left(h^{2}=0.30-0.34\right.$ for sweet taste perception; Hwang et al., 2015; $h^{2}=0.47-0.90$ for BMI; Elks et al., 2012). Recent genome-wide association studies (GWAS) of BMI identified an enrichment of associated genetic variants involved in the central nervous system (CNS) that might be linked to sweet taste perception. These include genetic variants in loci associated with insulin, glucose, and adipogenesis regulation (Locke et al., 2015), as well as energy balance, regulation of appetite, and food intake preferences (Speliotes et al., 2010). However, it is still unclear whether some genes are jointly responsible for both the regulation of sweet taste perception and BMI.

In the present study, we used longitudinal and genetic data from a sample of 1,576 young Australian twins to investigate the relationship between sweet taste and BMI. We first examined the association between sweet taste perception and BMI, both measured during adolescence. Next, we examined whether adolescent taste perception predicted BMI at a later time in life (9 years later). Finally, we assessed the genetic overlap between BMI and sweet perception using twin modeling and polygenetic risk scores approaches to determine whether the association between the two traits was due to shared genetic components.

\section{Materials and Methods \\ Data}

Participants were adolescent and young adult twins and their singleton siblings from the Brisbane Adolescent Twin Study (Wright \& Martin, 2004), also referred to as the Brisbane Longitudinal Twin Study (BLTS). They completed a taste test at around $15.8 \pm 2.6$ years old and had their BMI measured at the clinic (two thirds) or self-reported (one third). They reported their BMI again approximately 9 years later as part of a follow-up study. Descriptive statistics for the participants are displayed in Table 1 . The study was conducted in accordance with the Declaration of Helsinki and informed consent was obtained from all adult participants and from parents for participants under age of 18 years. Approval for this study was obtained from the Human Research Ethics Committee of QIMR Berghofer Medical Research Institute.

The taste test included 10 different solutions, of which five were bitter, four were sweet (described below), and one was neutral (i.e., water, as control; Hwang et al., 2015). The four sweet solutions included two sugars $(0.60 \mathrm{M}$ glucose

\begin{tabular}{|c|c|c|}
\hline \multicolumn{3}{|c|}{$\begin{array}{l}\text { Baseline Characteristics of Participants According to } \\
\text { Taste Perception }\end{array}$} \\
\hline & Early age (12-26) & Later age (18-38) \\
\hline$N$ of participants & 1,576 & 998 \\
\hline MZ pairs & 205 & 101 \\
\hline DZ pairs & 395 & 197 \\
\hline Siblings & 376 & 402 \\
\hline Age (years) & $15.8 \pm 2.6$ & $25.2 \pm 4$ \\
\hline Female & $53.5 \%$ & $61.1 \%$ \\
\hline Height $(\mathrm{cm})$ & $162.7 \pm 8.2$ & $171.8 \pm 9.7$ \\
\hline Weight (kg) & $54.9 \pm 11.6$ & $70.9 \pm 15.1$ \\
\hline $\operatorname{BMI}\left(\mathrm{kg} / \mathrm{m}^{2}\right)$ & $20.6 \pm 3.5$ & $23.8 \pm 4.0$ \\
\hline \multicolumn{3}{|l|}{ Intensity rating } \\
\hline Glucose & $31.2 \pm 15.7$ & \\
\hline Fructose & $31.9 \pm 17.6$ & \\
\hline NHDC & $34.4 \pm 18.6$ & \\
\hline Aspartame & $26.3 \pm 16.0$ & \\
\hline gSweet & $31.1 \pm 15.0$ & \\
\hline \multicolumn{3}{|c|}{$\begin{array}{l}\text { Note: Data are presented as mean } \pm \text { standard deviation. } \\
\text { Intensity ratings are millimeter on a labeled magni- } \\
\text { tude scale. NHDC = neohesperidine dihydrochalcone. } \\
\text { gSweet }=\text { general sweet intensity. }\end{array}$} \\
\hline
\end{tabular}

and $0.30 \mathrm{M}$ fructose) and two non-caloric sweeteners (8.0 $\times 10^{-5} \mathrm{M} \mathrm{NHDC}$ and $1.4 \times 10^{-3} \mathrm{M}$ aspartame). Each solution and the water control were presented twice (i.e., total of 20 solutions) in color-coded $2 \mathrm{~mL}$ polypropylene microcentrifuge tubes with flip tops. The first 10 tubes contained one presentation of each compound plus the water control and the next 10 contained the same solutions in a different order. The order of all 20 tubes was the same for all participants. Participants were instructed to: (1) open the tube, swish the solution in the mouth for $5 \mathrm{~s}$, and spit out; (2) rate the perceived intensity of the solution; (3) rinse the mouth out four times with tap water; and (4) repeat steps 1 to 4 for each tube. Perceived intensity was rated on a general labeled magnitude scale (gLMS; Green et al., 1993) with labels of no sensation $(0 \mathrm{~mm})$, barely detectable $(2 \mathrm{~mm})$, weak $(7 \mathrm{~mm})$, moderate $(20 \mathrm{~mm})$, strong $(40 \mathrm{~mm})$, very strong $(61 \mathrm{~mm})$, and strongest imaginable $(114 \mathrm{~mm})$. Participants marked a line on the scale where they thought the sensation fitted. The mean intensity ratings from the duplicate presentations were used in this study. As our previous study showed that a common genetic component accounted for most of the variance in intensity scores of each sweetener ( $71 \%$ for glucose, $77 \%$ for fructose, $64 \%$ for NHDC, and $59 \%$ for aspartame; Hwang et al., 2015), here we calculated a general sweet intensity rating (gSweet) using the weighted mean of intensity ratings of the four sweeteners. Intensity scores were square-root transformed to approximate the normal distribution.

\section{Genotyping Quality Control}

Participants were genotyped using the IlluminaHuman610W-Quad bead chip. Standard quality control was performed on genotyped variants: single nucleotide polymorphism (SNP) with call rate $<0.95$, deviation from Hardy-Weinberg equilibrium (HWE) $p$ value $<10^{-6}$, or 
minor allele frequency (MAF) $<0.01$ were excluded (Medland et al., 2009). To control for population stratification, we excluded individuals which lay beyond six standard deviations from the first two genotypic principal components centroid of the 1,000 Genome European descent populations. Imputation was carried out based on the August 4, 2010 version of the publicly released 1,000 Genomes Project European genotypes using MACH (Li et al., 2010). We implemented a filtering threshold of 0.3 on the $r^{2}$ metric for each of the SNPs in line with recommended practice in $\mathrm{MACH}$, and a MAF $>0.01$.

\section{Twin Modeling}

Phenotypic and genetic correlations between measures of taste intensity and BMI were estimated using bivariate variance components modeling in the structural equation software package $\mathrm{Mx}$, which utilizes maximum likelihood estimation procedures (Neale \& Cardon, 1992). Variance components modeling partitions the variation of a trait into genetic and environmental sources by leveraging the degree of genetic differences between monozygotic twins (MZ; share all genes) and dizygotic twins (DZ; share half of genes) pairs. These known differences allow the estimation of additive genetic (A), common environment (C), and unique environment (E, which also includes experimental error and random noise) parameters in a variance components model (ACE). The comparative fit of models was assessed by calculating the Log-likelihood Ratio Test (LRT) statistics (Wilks, 1938). All the models were adjusted for sex and the participants' corresponding age.

\section{Polygenic Risk Scores}

To complement the genetic correlation estimates, we also carried out a polygenic risk prediction analysis by computing polygenic risk scores (PGRS) of our trait of interest (in this case, BMI) and then using this genetic profile to predict another trait (i.e., taste perception) (International Schizophrenia Consortium et al., 2009). In short, the PGRS describes predicted phenotypic values that are genetically derived. It is computed by aggregating the estimated effects of many variants multiplied by the number of observed effect allele into a single score for each individual, to mimic a genetic proxy (profile scoring) for the underlying phenotypic trait. In our analysis, we used the association estimates from the latest GWAS meta-analysis summary statistics of BMI from the Genetic Investigation of Anthropometric Traits (GIANT) consortium including up to 339,225 participants (Locke et al., 2015). We extracted effect estimates of the SNPs that were computed based on participants $(n=322,154)$ of European ancestry only. This approach could yield increased power to detect genetic correlations due to the large sample size of the GIANT analysis. Given that the samples in this study were also part of the GIANT BMI GWAS, these GWAS estimates were recomputed after removing the overlapping samples. Next, we selected variants to compute the PGRS based on 10 different $p$-value thresholds $(<.00001, .001, .01, .05, .1, .2$, $.3, .4, .5, .75)$. Linkage disequilibrium (LD) clumping was performed to remove redundant SNPs $\left(r^{2}>0.2\right)$ within a $500 \mathrm{~kb}$ window for each of the variants in the PGRS. The computation of PGRS and LD clumping were carried out using PLINK (Chang et al., 2015) by multiplying the number copies of each effect allele with the reported magnitude of association and summing over all the relevant SNPs. Ambiguous SNPs with complementary strands (A/T, G/C) were removed. Specifically, the number of independent genetic variants for each of the thresholds was 2,071, 8,403, $27,638,79,871,136,195,237,876,333,747,424,237$, and 511,295 SNPs. Finally, we converted each of the PGRS to standard $z$ scores $(\mu=0$ and $\sigma=1)$ and tested their association with the different sweet taste perception scores using a linear mixed model, fitting the first three principal components derived from the genotypes, age, and sex as covariates, and the family ID as random effect.

The polygenic risk prediction approach carries a high multiple testing burden given that each of the PGRS is tested for their association with sweet taste perception. However, given that the different sweet taste perception are correlated between each other and that each of the PGRS are highly redundant, we estimated the number of independent tests using a matrix spectral decomposition algorithm (Nyholt, 2004). This algorithm estimates the equivalent number of independent variables from a correlation matrix, by examining the ratio of observed eigenvalue variance to its theoretical maximum. Using this, we estimated the number of independent test to be 7 , thus our bonferroni-corrected significance threshold was $p=.05 / 7=.007$.

\section{Results}

We investigated whether sweet taste perception in adolescence was predictive of BMI in adolescence (15.8 \pm 2.6 years old) and early adulthood (25.2 \pm 4.0 years old). In our regression analyses, we found an association between the perceived intensity of sweetness and BMI at the same age in adolescence, with glucose and aspartame showing significant correlations of $r=0.056(p=.035)$ and $r=0.058(p$ $=.029)$, respectively (Table 2 ). When investigating whether sweet taste perception was predictive of early adulthood $\mathrm{BMI}$, we found stronger associations with all sweet perception scores ranging from $r=0.07$ for fructose to $r=0.09$ for aspartame (Table 2). Intensity ratings of water were used as a negative control, and, as expected, they were not associated with BMI.

Using twin modeling, we found a positive genetic correlation between perceived intensity of all the tastes and BMI at same and later age. However, only the perceived intensity of glucose showed a significant association (same age: $r_{\mathrm{g}}=$ $0.13, p=.03$; later age: $r_{\mathrm{g}}=0.21, p=.017$; Table 2 ). The 


\section{TABLE 2}

Summary of Phenotypic and Genetic Correlations Between Taste Intensity Ratings and BMI

\begin{tabular}{|c|c|c|c|c|}
\hline \multirow[b]{2}{*}{ Phenotypic correlations } & \multicolumn{2}{|c|}{ BMI same age } & \multicolumn{2}{|c|}{ BMI later age } \\
\hline & $r_{p} \pm S E$ & $p$ & $r_{p} \pm S E$ & $p$ \\
\hline gSweet & $.05 \pm .03$ & .059 & $.08 \pm .03$ & .012 \\
\hline Glucose & $.06 \pm .03$ & .035 & $.08 \pm .03$ & .020 \\
\hline Fructose & $.04 \pm .03$ & .196 & $.07 \pm .03$ & .034 \\
\hline NHDC & $.04 \pm .03$ & .122 & $.07 \pm .03$ & .025 \\
\hline Aspartame & $.06 \pm .03$ & .029 & $.09 \pm .03$ & .007 \\
\hline $\mathrm{H}_{2} \mathrm{O}$ & $-.02 \pm .03$ & .579 & $-.03 \pm .03$ & .406 \\
\hline Genetic correlations & $r_{g} \pm S E$ & $p$ & $r_{\mathrm{g}} \pm S E$ & $p$ \\
\hline gSweet & $.10 \pm .06$ & .092 & $.16 \pm .08$ & .053 \\
\hline Glucose & $.13 \pm .06$ & .030 & $.21 \pm .08$ & .017 \\
\hline Fructose & $.08 \pm .06$ & .173 & $.14 \pm .08$ & .150 \\
\hline NHDC & $.07 \pm .06$ & .260 & $.10 \pm .08$ & .267 \\
\hline Aspartame & $.12 \pm .06$ & .077 & $.18 \pm .08$ & .090 \\
\hline $\mathrm{H}_{2} \mathrm{O}$ & $.00 \pm .06$ & .955 & $-.12 \pm .08$ & .172 \\
\hline
\end{tabular}

Note: All values are calculated using bivariate variance components modeling in the structural equation software package Mx. Genetic correlations are calculated from bivariate $A E$ model as $C$ components can be dropped without worsening the model fit $(p=.28-.87$ for each trait). gSweet $=$ general sweet intensity. $\mathrm{NHDC}=$ neohesperidine dihydrochalcone. Estimates in bold are statistically different from zero.

environmental correlations were not significantly different from zero (data not shown).

To further assess the genetic overlap between BMI and sweet taste perception, we carried out a polygenic risk prediction analysis. Based on the GWAS of BMI from the GIANT consortium, we computed individual BMI PGRS, including a different number of variants based on $p$-value thresholds, and tested their association with sweet taste perception and BMI itself. As anticipated, each of the PGRS were strongly associated with BMI (e.g., the PGRS based on SNPs with a $p$ value $<.2$ in the GIANT GWAS had an $r=0.2 ; p<1.0 \times 10^{-15} ;$ Figure S1). Through this approach, we found a significant genetic correlation between BMI and NHDC (Figure 1) but not with glucose, as with the twin modeling analysis.

\section{Discussion}

We found that the perceived intensity of sweet solutions measured during adolescence was positively associated with BMI measured at the same age, and that the association was stronger with BMI measured 9 years later. We also found evidence of a positive genetic correlation of glucose and NHDC with BMI.

In contrast to findings from previous cross-sectional studies, which showed negative associations (Bartoshuk et al., 2006; Overberg et al., 2012) or no associations between sweet taste perception and BMI (Anderson, 1995; Grinker, 1978; Joseph et al., 2016), we found a subtle but positive association with BMI measured at the same age. The differences may result from variation in study designs and sample demographics. Bartoshuk et al. (2006) reported a negative association between perceived sweetness of a candy and BMI in a large sample of college students $(n=3,700)$, whereas the taste measures in our study were adolescents' intensity ratings of sweet solutions. Overberg et al. (2012) collected data from adolescents as well as children (age ranged from 6 to 18 years) and they reported a negative association by dichotomizing participants into either obese (BMI $>$ 97th percentile) or normal weight (BMI $<90$ th percentile). In our study, BMI was defined as a continuous variable, which provided us more robust estimates with respect to the magnitude and direction of the correlation. In addition, studies (Anderson, 1995; Grinker, 1978; Joseph et al., 2016) that showed no association typically have relatively small sample sizes $(n<100)$, which suggests that larger samples are necessary to detect a significant association.

Our finding that sweet taste perception has a higher association with BMI at a later age (as compared to BMI obtained at the same time of taste perception measurement) yields several possible explanations. The first could be the cumulative effect of sweet preferences. Individual differences in sweet perception may gradually contribute to a bigger variation in preferences for sweetness, which directly influence sweet food consumption, and further lead to a bigger variation in BMI later in life. Second, during adolescence, diet preferences are strongly influenced by cultural practice and familial influences (Scaglioni et al., 2008), rather than the true taste preferences. Also, BMI at a young age is susceptible to body and hormonal changes rather than sweet food consumption, compared to that in a later age when body mass change becomes more stable.

Our two genetic approaches independently identified significant correlations between BMI and sweet taste perception; one with glucose and the other with NHDC. Nevertheless, these correlations were all positive regardless of taste stimuli and the underlying modeling approaches. In the context of our study, the twin modeling and PGRS analyses complement each other in the sense that the PGRS models the cross-trait genetic overlap based on common genetic variants, while conventional twin models incorporate a larger spectrum of information, including undiscovered causal SNPs and rare variants, in determining coheritability.

Our genetic overlap observation is consistent with the biological literature. Simple carbohydrates (sugars) as well as complex carbohydrates (oligosaccharides and polysaccharides) are the main source of energy for the human body, and over-consumption of these sweet compounds can result in weight gain as excess energy intake is transformed and stored as body fat (Aarsland et al., 1997). While the GIANT consortium's analysis identified several BMIassociated genes with putative functions in the brain, some of those genes could be responsible for sweet taste perception, such that genetic variants might affect BMI by influencing dietary behaviors. Similarly, there is some evidence that individual differences in taste responses are due to differences in signal processing in the CNS (Green \& 

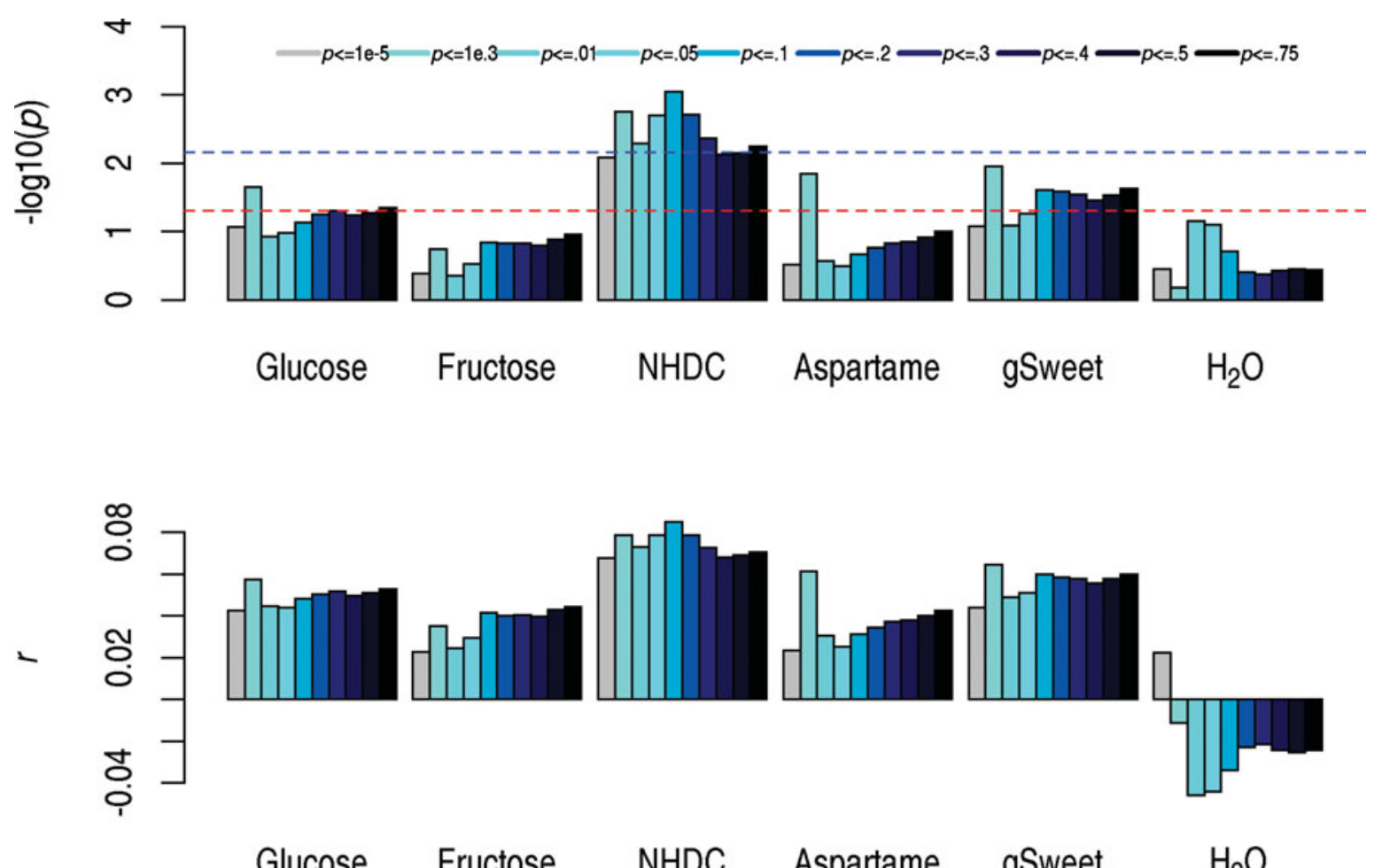

\section{FIGURE 1}

(Colour online) Results of the polygenic risk prediction. Note: The bars correspond to the association between each of the polygenic risk scores (PGRS) of BMI computed based on the specified $p$-value thresholds $(<.00001, .001, .01, .05, .1, .2,0.3,0.4,0.5,0.75)$ and the different sweet taste intensity scores. $p$ values of the association are shown in the logarithmic scale in the upper part of the figure while spearman correlations $(r)$ are shown in the bottom part. Red dotted line shows the nominal $p$-value (.05) on -log10 scale. Blue dotted line shows the significance threshold after accounting for multiple testing. As an example, the first bar should be interpreted as the correlation between a genetically predicted BMI value based on SNPs with a $p$ value $<1.0 \times 10^{-5}$ in the GIANT BMI GWAS and glucose. gSweet $=$ general sweet intensity. NHDC = neohesperidine dihydrochalcone.

George, 2004). Other pathways may also contribute to explaining the genetic overlap between sweet perception and BMI. For example, the neurobiology of food reward might help explain why the use of non-caloric sweeteners can result in weight gain without affecting calorie intake (Heid et al., 2010). Lastly, apart from the neurological and other possible mechanisms explained above, sweet taste receptors in the mouth or guts can also control metabolism through various mechanisms, such as influencing insulin secretion (Kyriazis et al., 2014; Laffitte et al., 2014). Regardless of the scenario, sweet taste perception appears to play an important role in regulating BMI. However, in this study, we were not able to prove the directionality of this association.

The strengths of our study included that the twin data allowed us to estimate the genetic and environmental contributions to the correlation between sweet taste and BMI. Also, the PGRS of BMI derived from common variants provided further support for the genetic correlation between the two and made our findings more robust. Lastly, the longitudinal data for BMI measured 9 years apart allowed us to test the long-term effects of sweet taste perception on BMI. We must also acknowledge some limitations; for example, the effective sample size $\left(N_{\text {same age }}=1,576\right.$ and $N_{\text {later age }}=$ 998) could be considered small for a twin study, and the use of self-reported BMI might potentially introduce some modest bias, as it has been pointed out that participants tend to slightly overestimate their height and underestimate their weight (Reed \& Price, 1998).

In conclusion, we showed that adolescent sweet taste perception in adolescence is associated with BMI at both adolescence and early adulthood, and that this association is partly explained through their genetic overlap. Identification of the shared genetic architecture could improve our understanding of the genetic pathways underlying both sweet taste perception and weight gain. As we obtain more study samples in the future, we will use the SNPs that strongly associate with sweet taste perception as genetic instruments to make causal inference (i.e., Mendelian randomization studies) about the effect of sweet taste perception on weight gain and diet-related disorders, which may provide insights into their prevention and treatment. 


\section{Acknowledgments}

We thank Kirsten J. Mascioli, Christopher Tharp, Fujiko Duke, Deborah Lee, and Corrine Mansfield from the Monell Chemical Senses Center for manufacturing the taste tests; and from the QIMR Berghofer, Marlene Grace, Ann Eldridge, Natalie Garden, Kerrie McAloney for project coordination, data collection, and data entry, and David Smyth and Anthony Conciatore for computer support. In particular, thanks go to twins and their families for their participation. This work was supported by the National Institute of Health, grants DC02995 to PASB and DC004698 and DC011735 to DRR and the Australian NHMRC grants 241944 and 1031119 to NGM. LH receives scholarship support from QIMR Berghofer Medical Research Institute. GCP receives scholarship support from the University of Queensland and QIMR Berghofer Medical Research Institute. MER received support from the NHMRC Centre for Research Excellence on Suicide Prevention [GNT1042580].

\section{Conflict of Interest}

The authors declare that they have no conflict of interest.

\section{Supplementary Material}

To view supplementary material for this article, please visit http://dx.doi.org/10.1017/thg.2016.60.

\section{References}

Aarsland, A., Chinkes, D., \& Wolfe, R. R. (1997). Hepatic and whole-body fat synthesis in humans during carbohydrate overfeeding. American Journal of Clinical Nutrition, $65,1774-1782$.

Anderson, G. H. (1995). Sugars, sweetness, and food intake. American Journal of Clinical Nutrition, 62, 195S-201S; discussion 201S-202S.

Bartoshuk, L. M., Duffy, V. B., Hayes, J. E., Moskowitz, H. R., \& Snyder, D. J. (2006). Psychophysics of sweet and fat perception in obesity: Problems, solutions and new perspectives. Philosophical Transactions of the Royal Society of London, Series B, Biological Sciences, 361, 1137-1148.

Björntorp, P. (1995). Endocrine abnormalities of obesity. Metabolism, 44, 21-23.

Breslin, P. A., \& Spector, A. C. (2008). Mammalian taste perception. Current Biology, 18, R148-155.

Chang, C. C., Chow, C. C., Tellier, L. C., Vattikuti, S., Purcell, S. M., \& Lee, J. J. (2015). Second-generation PLINK: Rising to the challenge of larger and richer datasets. Gigascience, 4, 7.

Elks, C. E., den Hoed, M., Zhao, J. H., Sharp, S. J., Wareham, N. J., Loos, R. J., \& Ong, K. K. (2012). Variability in the heritability of body mass index: A systematic review and metaregression. Frontiers in Endocrinology (Lausanne), 3, 29.

Fox, K. R., \& Hillsdon, M. (2007). Physical activity and obesity. Obesity Reviews, 8, 115-121.
Green, B. G., \& George, P. (2004). 'Thermal taste' predicts higher responsiveness to chemical taste and flavor. Chemical Senses, 29, 617-628.

Green, B. G., Shaffer, G. S., \& Gilmore, M. M. (1993). Derivation and evaluation of a semantic scale of oral sensation magnitude with apparent ratio properties. Chemical Senses, $18,683-702$.

Grinker, J. (1978). Obesity and sweet taste. American Journal of Clinical Nutrition, 31, 1078-1087.

Heid, I. M., Jackson, A. U., Randall, J. C., Winkler, T. W., Qi, L., Steinthorsdottir, V., ... Lindgren, C. M. (2010). Meta-analysis identifies 13 new loci associated with waist-hip ratio and reveals sexual dimorphism in the genetic basis of fat distribution. Nature Genetics, 42, 949-960.

Hwang, L. D., Zhu, G., Breslin, P. A., Reed, D. R., Martin, N. G., \& Wright, M. J. (2015). A common genetic influence on human intensity ratings of sugars and high-potency sweeteners. Twin Research and Human Genetics, 18, 361367.

Joseph, P. V., Reed, D. R., \& Mennella, J. A. (2016). Individual differences among children in sucrose detection thresholds: Relationship with age, gender, and bitter taste genotype. Nursing Research, 65, 3-12.

Kyriazis, G. A., Smith, K. R., Tyrberg, B., Hussain, T., \& Pratley, R. E. (2014). Sweet taste receptors regulate basal insulin secretion and contribute to compensatory insulin hypersecretion during the development of diabetes in male mice. Endocrinology, 155, 2112-2121.

Laffitte, A., Neiers, F., \& Briand, L. (2014). Functional roles of the sweet taste receptor in oral and extraoral tissues. Current Opinion in Clinical Nutrition and Metabolic Care, 17, 379-385.

Li, Y., Willer, C. J., Ding, J., Scheet, P., \& Abecasis, G. R. (2010). $\mathrm{MaCH}$ : Using sequence and genotype data to estimate haplotypes and unobserved genotypes. Genetic Epidemiology, 34, 816-834.

Locke, A. E., Kahali, B., Berndt, S. I., Justice, A. E., Pers, T. H., Day, F. R., ... Speliotes, E. K. (2015). Genetic studies of body mass index yield new insights for obesity biology. $\mathrm{Na}$ ture, 518, 197-206.

Masubuchi, Y., Nakagawa, Y., Ma, J., Sasaki, T., Kitamura, T., Yamamoto, Y., ... Shibata, H. (2013). A novel regulatory function of sweet taste-sensing receptor in adipogenic differentiation of 3T3-L1 cells. PLoS One, 8, e54500.

Medland, S. E., Nyholt, D. R., Painter, J. N., McEvoy, B. P., McRae, A. F., Zhu, G., ... Martin, N. G. (2009). Common variants in the trichohyalin gene are associated with straight hair in Europeans. American Journal of Human Genetics, 85, 750-755.

Moskowitz, H. R., Kluter, R. A., Westerling, J., \& Jacobs, H. L. (1974). Sugar sweetness and pleasantness: Evidence for different psychological laws. Science, 184, 583-585.

Neale, M., \& Cardon, L. (1992). Methodology for genetic studies of twins and families. Dordrecht: Kluwer Academic Publishers B.V.

Nyholt, D. R. (2004). A simple correction for multiple testing for single-nucleotide polymorphisms in linkage 
disequilibrium with each other. American Journal of $\mathrm{Hu}$ man Genetics, 74, 765-769.

Overberg, J., Hummel, T., Krude, H., \& Wiegand, S. (2012). Differences in taste sensitivity between obese and nonobese children and adolescents. Archives of Disease in Childhood, 97, 1048-1052.

International Schizophrenia Consortium, Purcell, S. M., Wray, N. R., Stone, J. L., Visscher, P. M., O’Donovan, M. C., ... Sklar, P. (2009). Common polygenic variation contributes to risk of schizophrenia and bipolar disorder. Nature, 460 , 748-752.

Reed, D. R., \& Price, R. A. (1998). Estimates of the heights and weights of family members: Accuracy of informant reports. International Journal of Obesity and related Metabolic Disorders, 22, 827-835.

Rolls, E. T. (2015). Taste, olfactory, and food reward value processing in the brain. Progress in Neurobiology, 127-128, 6490.

Scaglioni, S., Salvioni, M., \& Galimberti, C. (2008). Influence of parental attitudes in the development of children eating behaviour. British Journal of Nutrition, 99, S22-25.
Solomon, C. G., \& Manson, J. E. (1997). Obesity and mortality: A review of the epidemiologic data. American Journal of Clinical Nutrition, 66, S1044S-S1050.

Speliotes, E. K., Willer, C. J., Berndt, S. I., Monda, K. L., Thorleifsson, G., Jackson, A. U., ... Loos, R. J. (2010). Association analyses of 249,796 individuals reveal 18 new loci associated with body mass index. Nature Genetics, 42, 937948.

Wardle, J. (2007). Eating behaviour and obesity. Obesity Reviews, 8, 73-75.

Wilks, S. S. (1938). The Large-sample distribution of the likelihood ratio for testing composite hypotheses. Annals of Mathematical Statistics, 9, 60-62.

Wisse, B. E. (2004). The inflammatory syndrome: The role of adipose tissue cytokines in metabolic disorders linked to obesity. Journal of the American Society of Nephrology, 15, 2792-2800.

Wright, M. J., \& Martin, N. G. (2004). Brisbane adolescent twin study: Outline of study methods and research projects. Australian Journal of Psychology, 56, 65-78. 